(C) 2016 IEEE. Personal use of this material is permitted. Permission from IEEE must be obtained for all other uses, in any current or future media, including reprinting/republishing this material for advertising or promotional purposes, creating new collective works, for resale or redistribution to servers or lists, or reuse of any copyrighted component of this work in other works. 


\section{Multi-Functional Composite Metamaterial-Inspired EEAD Antenna for Structural Applications}

\author{
Thomas C. Baum and Kamran Ghorbani \\ School of Electrical and Computer Engineering \\ RMIT University, \\ Melbourne, Australia \\ thomas.baum@rmit.edu.au
}

\author{
Amir Galehdar \\ Maritime Division \\ DSTG - Fisherman's Bend \\ Melbourne, Australia \\ amir.galehdar@dsto.defence.gov.au
}

\author{
Kelvin J. Nicholson \\ Aerospace Composite Technologies \\ DSTG - Fisherman's Bend \\ Melbourne, Australia \\ kelvin.nicholson@dsto.defence.gov.au
}

\author{
Richard W. Ziolkowski \\ Department of Electrical and Computer Engineering \\ University of Arizona \\ Tucson, AZ, USA \\ ziolkowski@ece.arizona.edu
}

\begin{abstract}
An electrically small, load-bearing Egyptian axe dipole (EAD) antenna has been sewn into a low loss, pure quartz glass composite material to investigate its performance. Previous investigations of embroidered Egyptian axe dipole antennas indicated that the dielectric losses of the associated epoxy-based composite, in conjunction with the high effective surface resistance of the conductive textile threads, significantly degrade their performance. Simulations of the EAD antenna using a composite sandwich structure based on an advanced embroidery technique and the much lower loss quartz fabric have shown that a realized gain of $0.9 \mathrm{dBi}$ is possible, a dramatic improvement over previous realizations.
\end{abstract}

Keywords-Composite structures, electrically small antennas, glass fiber reinforced polymer, metamaterial-inspired

\section{INTRODUCTION}

The recent popularity of embroidered textiles circuits and sensors has led to the development of novel wearable devices [1-5]. Wearable textiles have found a unique home in biomedical, health and sensory applications [1-5]. The transition of these simple digital or DC circuits to microwave devices (such as antennas) has been less successful from a performance perspective. This is due to the high losses associated with commercial conductive threads and the dimensional variability of a textile substrate [6]. Investigations of conformal composite antennas have been used to mitigate the dimensional variability seen in wearable textiles by encapsulating RF devices (such as antennas) in an epoxy resin [7]. Extending this further, the development of conformal loadbearing antenna structures (CLAS) provides a unique opportunity to incorporate conductive textiles into load-bearing structures [8]. To realize truly conformal CLAS, embroidery techniques are employed to create the appropriate conductive patterns. The structural capacity of the substrate can be increased by using a class of compose materials called prepregs. These pre-pregs consist of a fiber matrix such as glass, Kevlar or carbon fiber which are pre-impregnated with a precision amount of toughened resin to achieve the best possible fiber-volume fraction [9]. For electrical devices the choice of pre-preg is restricted to Kevlar or glass as they are both dielectric materials.

The implementation of embroidered CLAS can be enhanced by utilizing electrically small antennas (ESAs). ESAbased CLAS are well adapted to unmanned aerial systems (UAS) due to their small size. One of the drawbacks with ESAs, however, is the traditional tradeoff in their bandwidth and gain performances with the associated decrease in size. Nonetheless, their bandwidths can be enhanced significantly by augmenting them with active elements, such as negative impedance converters (NIC) [10], to control their input impedances. In addition to these fundamental limitations of ESAs, their radiation efficiency performance is highly dependent on the use of low loss substrates. In particular, because the electromagnetic fields they generate are highly concentrated along and around their conductors, the performance characteristics of ESAs are sensitive to the use of lossy materials to construct them. This makes them less attractive as an embroidered antenna as there are also further losses incurred from the conductive threads [8]. The realization of a functional ESA antenna using embroidery techniques poses numerous challenges.

This paper provides an investigation of an alternative structural sandwich composite panel incorporating an electrically small, near-field resonant parasitic (NFRP) Egyptian axe dipole (EAD) topology [11]. Dielectric and conductive losses have been mitigated by combining an advanced embroidery technique with a low loss pure quartz fabric (Astro-Quartz II) and a low loss Cynate Ester resin (PT30). Both of these materials provide the lowest loss combination of all semi-structural composite materials certified for use in aerospace structures. We refer to the antenna as an Embroidered Egyptian Axe Dipole (EEAD) antenna. This work expands on previous work carried out by the authors in [8]. This previous work demonstrated that a NFRP EEAD 
antenna could be manufactured within a pre-preg. However, the dielectric and conductive losses in that work were found to be high, significantly decreasing its radiation efficiency. This paper explores the effects of conductive losses and the embroidery of the threads into pre-pregs for this type of ESA to create a much higher performance NFRP EEAD antenna.

\section{THE DEVELOPMENT OF AN NFRP EEAD ANTENNA}

All pre-preg materials consist of two material phases. These are a fiber phase and a host matrix phase, the latter consisting of a toughened resin. The advantage of a composite material over traditional materials, such as metals, is that they provide significantly higher strength to weight ratios compared to metals. Other benefits include good fatigue resistance and little thermal expansion. These attributes are also desirable for antenna structures. However, the fiber phase of a composite can consist of many different materials. Most aerospace structures deal with three primary materials: glass, Kevlar and carbon fiber. All of these fibers are typically woven into a cloth or fabrics which are impregnated with a toughened polymer resin matrix. The strength of a composite panel is highly linked to its fiber to resin volume fraction. The fundamental limit of these fractions for most typical structures is approximately $60 \%$ fiber to $40 \%$ resin. If dry cloth is processed under a technique call wet layup (i.e., resin is added to dry cloth), the control of the volume fraction is difficult. To prevent variability in the manufacture of structural panels, preimpregnated or pre-preg materials are used to give strict control over the final fiber volume fraction. Pre-preg materials are mostly stored within a freezer to extend their shelf life, prior to their implementation into a structure.

Treating pre-preg materials like any other cloth, modern computer controlled embroidery machines can be used to sew arbitrary patterns into them. From the perspective of developing CLAS-based systems, conductive threads can be sewn into pre-preg materials. This allows for a more streamlined manufacturing process, which can be carried out when incorporating these antennas into structural panels. The previous work by the authors [8] investigated an EEAD antenna sewn into a structural glass material (S-Glass) which was infused with a resin (HexPly 914E). This resin is an epoxy which has a high loss tangent $\left(\varepsilon_{\text {eff }}=4.265\right.$ and $\tan \delta=0.0194$ at $10 \mathrm{GHz}$ ). This antenna was designed with considerations for losses Simulated. The expected realized gain was approximated to be on the order of $0.5 \mathrm{dBi}$. The measured results, however, showed that the realized gain was on the order of $-5 \mathrm{dBi}$. As a result, the original loss model for the conductive thread (ShieldEx 100/34 dtex 2ply HC) was evaluated using comparative measurements of a sewn microstrip line.

This experimental investigation demonstrated that the original numerical model of the resistance of the sewn lines was quite inaccurate. The resistance of the lines was found to be a much more sophisticated issue. In fact, it was recognized that the conductive lines are, by their very nature, highly anisotropic conductors. This makes their modeling and characterization extremely complex. By analyzing various different stitching properties experimentally, the effective conductivity of the conductive threads was found to change significantly. To re-investigate the performance of the original
NFRP EEAD antenna, an updated model for the conductivity of the thread, which was derived from those measured results, has been included into the simulations to re-examine the behavior of the EEAD antenna reported in [8]. In particular, the approximate surface resistance at $300 \mathrm{MHz}$ was determined to be $\sim 0.14 \Omega /$ sq.

The simulated performance characteristics of the EEAD antenna with this updated model are summarized in Fig. 1. As shown in Fig. 1a, the maximum expected realized gain is -3.25 $\mathrm{dBi}$. While this outcome is still not perfect, it nevertheless gives a much better representation of the expected realized gain results. The significant deviation from the original results predicted by this updated model illustrates that there is a large variability and complexity in modeling the effective conductivity of the embroidered conductive lines. The values of the magnitude of the reflection coefficient, $\left|S_{11}\right|$, of the EEAD antenna model using two conductivity models of the thread as a function of the excitation frequency is given in Fig. 1b. It shows a significant difference between the performance of the EEAD antenna when a copper fiber or the ShieledEx fiber are used. Finally, for comparison, the corresponding measured $\left|S_{11}\right|$ values of the original EEAD antenna are provided in Fig. 2 for reference.

The results given in Fig. 1 demonstrate that the antenna is well matched to the $50 \Omega$ source at $0.295 \mathrm{GHz}$. Nonetheless, it
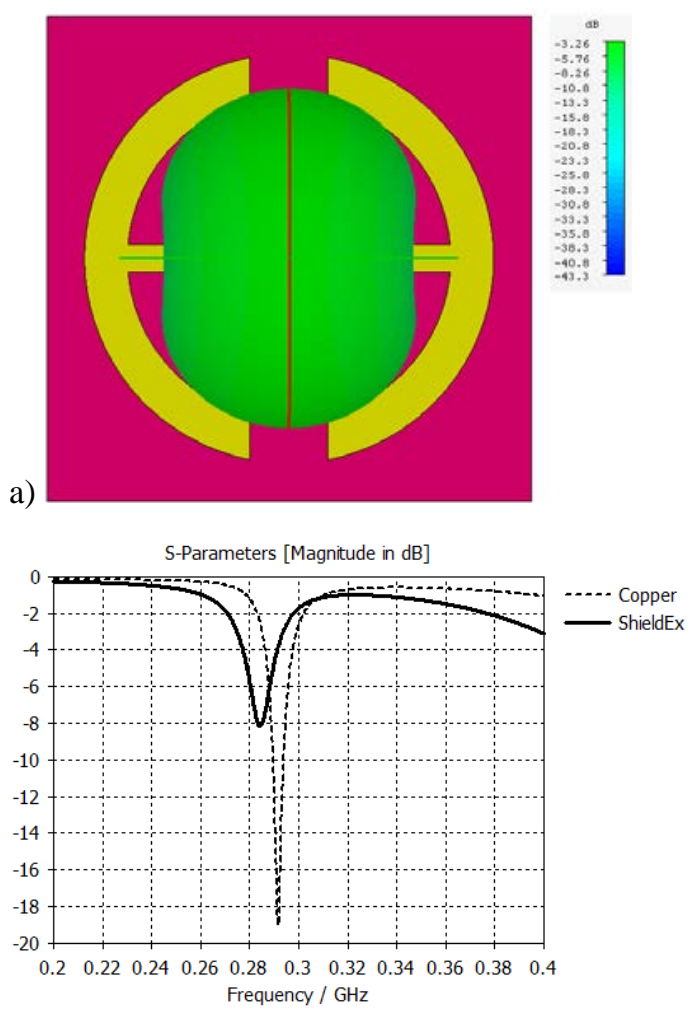

Fig. 1. Simulated performance characteristics of the original EEAD antenna in [8] with the updated conductivity model of the threads. a) Projected 3D farfield realized gain pattern at the resonance frequency $\sim 0.286 \mathrm{GHz}$, and b) simulated $\left|S_{11}\right|$ values as a function of the excitation frequency for the NFRP EEAD sewn with copper thread and the Shieldex thread. 


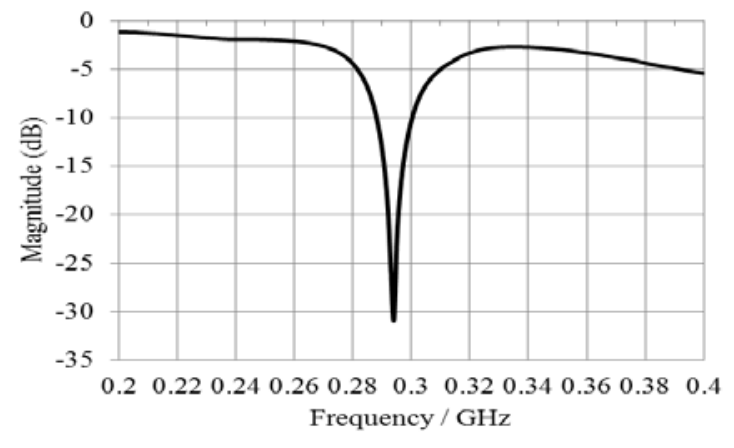

Fig. 2. Measured $\left|S_{11}\right|$ values as a function of the excitation frequency for the NFRP EEAD reported in [8].

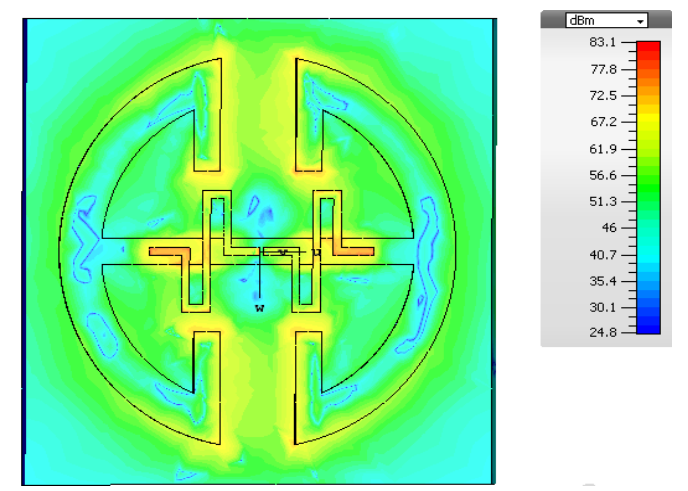

Fig. 3. Simulated loss density associated within the HexPly 914E substrate of the NFRP EEAD antenna.

is clear from the realized gain measurements that the power delivered to its terminals is being consumed by the dielectric and conductive losses in the materials. To analyze where these losses are occurring, a simulation of the loss density was carried out and is illustrated in Fig. 3. It demonstrates that the largest losses occur at the ends of the driven dipole element and at the corners and edges of the NFRP element. This is significant because the areas of highest loss occur at regions where the current must travel the furthest. These results confirm that the only real solution to mitigating these losses in the EEAD antenna is to increase the conductivity of the sewn thread and to reduce the dielectric losses within the substrate.

\section{LOW LOSS NFRP EEAD ANTENNA}

To combat the losses associated with the HexPly 914E substrate materials in [8], an advanced antenna substrate topology was investigated as a possible solution. Moving away from the original thick, single material substrate, an antenna design is introduced that consists of a high stiffness sandwich panel. Sandwich panels are commonly used on many aerospace structures where high stiffness and lightweight structures are required. An exploded view of this advanced topology is illustrated in Fig. 4a, while a consolidated side view of the final panel is illustrated in Fig $4 \mathrm{~b}$ to emphasize it thinness. The structure consists of three elements. The first is the embroidered NFRP feed elements and the EEAD antenna. The high loss ShieldEx fiber was replaced with a low loss Aracon XS0200E-025 Kevlar coated fiber. Under the same resistance measurements carried out for the ShieldEx fiber, the effective surface resistance of the Aracon fiber was found to be 0.02 $\Omega / \mathrm{sq}$ at $300 \mathrm{MHz}$. Another advantage that the Aracon fiber has over the ShieldEx fiber is its core fiber. The ShieldEx fiber core is a proprietary polyamide polymer, while the Aracon fiber is made from high strength Kevlar. The Kevlar core of the Aracon fiber can deliver a positive strength addition to a composite when compared to that of the ShielEx fiber.

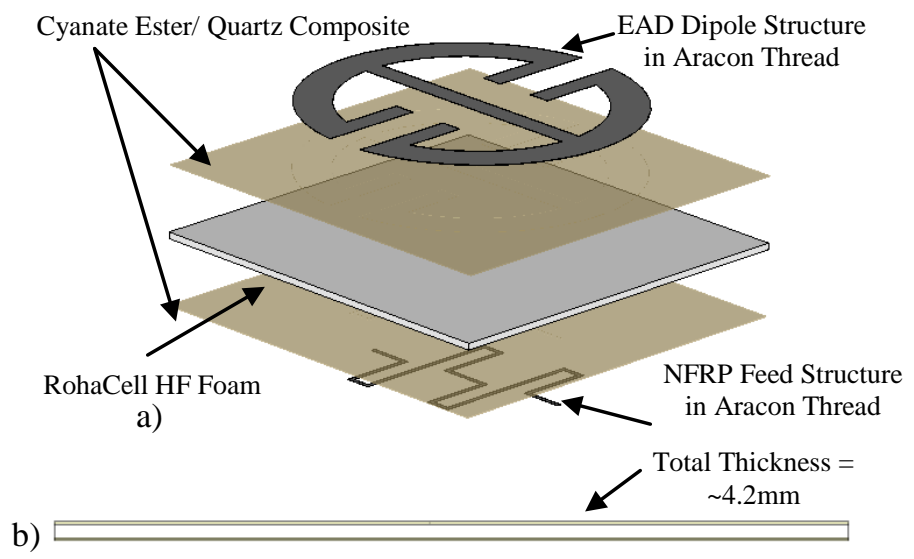

Fig. 4. NFRP EEAD antenna to be sewn with the low loss Cynate Ester resin and highly conductive Aracon thread a) An exploded rendering of the CST model, and b) side view of the consolidated EEAD antenna.

The second element of the advanced design is the introduction of an alternative low loss fabric made from a pure quartz material called Astro-Quartz. Astro-Quartz is a high strength fiber which is typically used within the aerospace industry for creating highly transparent RF windows such as radomes. The resin chosen to infuse into this composite is a low loss Cynate Ester resin. The combined composite has a measured relative dielectric constant of $\varepsilon_{\text {eff }}=3.31$ and a loss tangent equal to $\tan \delta=0.003$ at $10 \mathrm{GHz}$. This composite has a significantly lower loss than the HexPly 914E composite used in the original prototype EEAD Antenna.

The third and final element to help reduce the material losses is a foam core made from $3.0 \mathrm{~mm}$ of a low-loss Rohacell HF foam. Foams cores of this type are typically used in sandwich structures to reduce their weight. As indicated in Fig. $4 \mathrm{~b}$, the total combined thickness of the advanced panel is approximately $4.2 \mathrm{~mm}$.

The simulated results of the advanced antenna structure were also carried out with CST Microwave Studio using the best available and relevant material models. The projected 3D far-field realized gain pattern at the resonance frequency, 0.301 $\mathrm{GHz}$, is illustrated in Fig. 5a.

The simulated $\left|S_{11}\right|$ values as a function of the excitation frequency are given in Fig. 5b. As demonstrated by Fig. 5, the expected maximum realized gain value of this advanced EEAD antenna has been significantly improved. The realized gain is expected to be in the order of $0.905 \mathrm{dBi}$. While it is likely that this level of gain may not be achieved due to the complex nature of the sewn structures, this antenna topology nevertheless should be a significant step forward in 


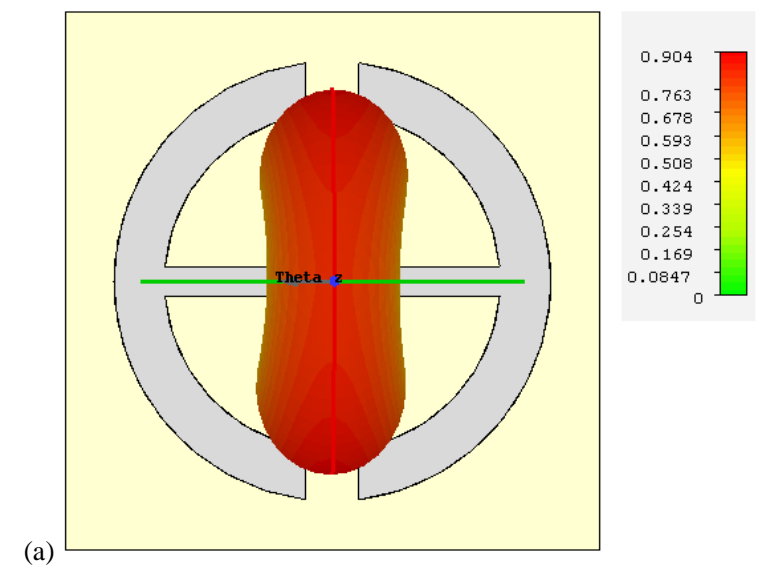

(a)

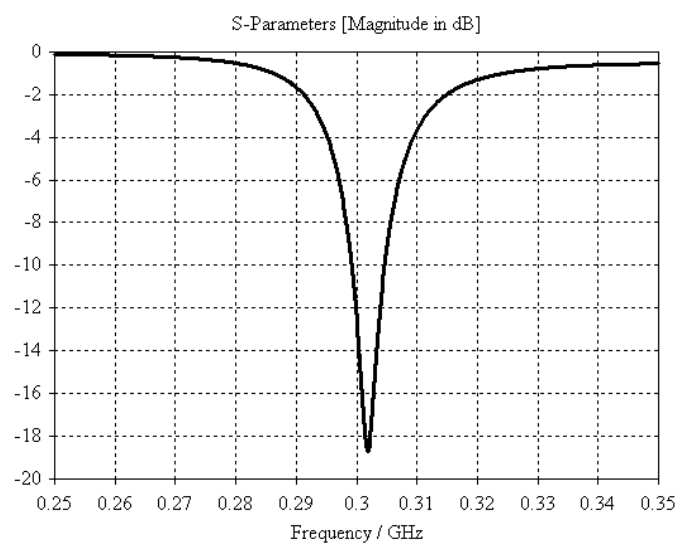

b)

Fig. 5. Simulated performance characteristics of the advanced NFRP EEAD antenna. a) Projected 3D far-field realized gain pattern at the resonance frequency $0.301 \mathrm{GHz}$, and $\mathrm{b}$ ) simulated $\left|\mathrm{S}_{11}\right|$ values as a function of the excitation frequency for the NFRP EEAD sewn with copper thread and the Shieldex thread

understanding how to create the best possible embroidered ESA antenna structures.

\section{MANUfACTURING THE EEAD ANTENNA}

The fabrication of the advanced NFRP EEAD antenna is currently taking place. Fig. 6 illustrates the current progress on the embroidery of the NFRP EEAD antenna structure into the Astro-Quartz fabric. Five layers of Astro-Quartz fabric are first stacked together and placed into an embroidery frame. To reduce warping within the fabric and to hold the five sheets together, a cross-hatched Kevlar pattern is then sewn into the Astro-Quartz fabric. Using a bobbin stitching technique the conductive fibers are then strapped into place with a virgin Kevlar thread. The Kevlar threads are those shown in Fig. 6, while the conductive threads are located on the opposite side of the fabric. If good performance of the alternative antenna topology is measured, it will provide a solid fundamental background for developing further and more complex EESAs for use as CLAS's. This may include Huygens source antennas or more complex 3D conformal ESA antennas [10-12].

Measured results of the alternative EEAD antennas structure will be reviewed and discussed in detail during our presentation.

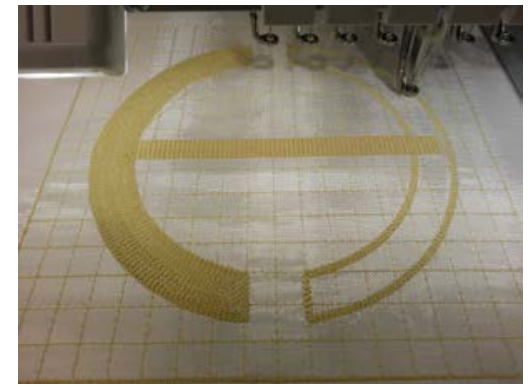

Fig. 6. Illustration of the advanced sandwitch panel NFRP EEAD antenna being manufactured

\section{ACKNOWLEDGMENT}

This work was supported by the Australian Department of Defense, Defense Science and Technology Group (DSTG), SRI-SME. The initial contributions by RWZ to this work occurred as the 2014-2015 Australian DSTO Fulbright Distinguished Chair in Advanced Science and Technology. He especially would like to thank the Australian-American Fulbright Commission and the Defense Sciences and Technology Group (formally DSTO) for their support.

\section{REFERENCES}

[1] D. Cottet, J. Grzyb, T. Kirstein, and G. Troster, "Electrical characterization of textile transmission lines," IEEE Trans. Advanced Packaging, vol. 26, no. 2, pp. 182-190, May 2003

[2] T. Linz, C. Kallmayer, R. Aschenbrenner, and H. Reichl, "Fully integrated EKG shirt based on embroidered electrical interconnections with conductive yarn and miniaturized flexible electronics," in Proc. International Workshop on Wearable and Implantable Body Sensor Networks, BSN 2006, Boston, MA, April 2006, pp. 4-26.

[3] T. Kaufmann and C. Fumeaux, "Wearable textile half-mode substrateintegrated cavity antenna using embroidered vias,” IEEE Antennas Wireless Propag. Lett., vol. 12, pp. 805-808, 2013.

[4] T. Kaufmann, D. C. Ranasinghe, M. Zhou, and C. Fumeaux, "Wearable quarter-wave folded microstrip antenna for passive UHF RFID applications,” Int. J. Antennas Propag., vol. 2013, Article ID 129839, 11 pages, 2013.

[5] G. Monti, L. Corchia, and L. Tarricone, "UHF wearable rectenna on textile materials," IEEE Trans. Antennas Propag., vol. 61, no. 7, pp. 3869-3873, Jul. 2013.

[6] R. Seager, S. Zhang, A. Chauraya, W. G. Whittow, Y. Vardaxoglou, T. Acti, and T. Dias, "Effect of the fabrication parameters on the performance of embroidered antennas," IET Microwaves Antennas Propag., vol. 7, no. 14, pp. 1174-1181, 2013.

[7] Z. Wang, L. Zhang, Y. Bayram, and J. L. Volakis, "Embroidered conductive fibers on polymer composite for conformal antennas," IEEE Trans. Antennas Propag., vol. 60, no. 9, pp. 4141-4147, Sep. 2012.

[8] K. J. Nicholson, T. C. Baum, K. Ghorbani and R. W. Ziolkowski, "Metamaterial-Inspired Electrically Small Antennas Integrated into Structural Materials,” in Proc. 2015 International Symposium on Antennas and propagation, Hobart, Australia, November 2015.

[9] A. A. Baker, S. Dutton and D. W. Kelly, Composite Materials for Aircraft Structures, 2nd Ed., American Institute of Aeronautics and Astronautics, Inc., Reston, VA, 2004.

[10] N. Zhu and R. W. Ziolkowski, "Broad-bandwidth, electrically small antenna augmented with an internal non-Foster element," IEEE Antennas Wireless Propag. Lett., vol. 11, pp. 1116-1120, 2012.

[11] R. W. Ziolkowski, P. Jin and C.-C. Lin, "Metamaterial-inspired engineering of antennas,” Proc. IEEE, vol. 99, no. 10, pp. 1720-1731, Oct. 2011.

[12] J. Peng and R. W. Ziolkowski, "Metamaterial-Inspired, Electrically Small Huygens Sources,” IEEE Antennas Wireless Propag. Lett., IEEE, vol. 9, pp. 501-505, 2010. 